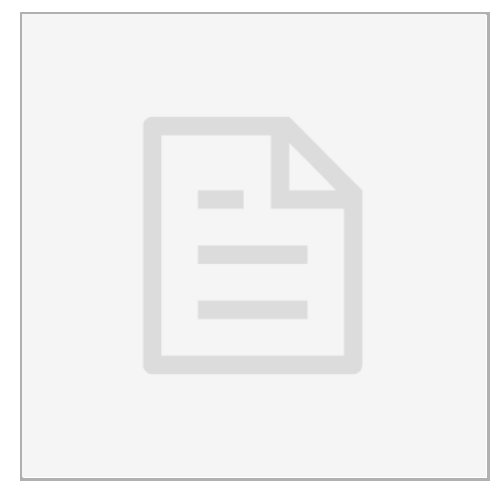

APR 05, 2019

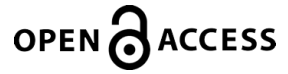

DOI:

dx.doi.org/10.17504/protocol s.io.zucf6sw

Protocol Citation: Sebastian ST Triesch 2019. Squalene Quantification using Nile Red Staining (Developmental). protocols.io

https://dx.doi.org/10.17504/p rotocols.io.zucf6sw

\section{License: This is an open} access protocol distributed under the terms of the Creative Commons Attribution License, which permits unrestricted use, distribution, and reproduction in any medium, provided the original author and source are credited

\section{Protocol status: In} development We are still developing and optimizing this protocol

Created: Apr 05, 2019

Last Modified: Apr 05, 2019

PROTOCOL integer ID:

22116

\section{(3) Squalene Quantification using Nile Red Staining} (Developmental)

V Forked from Squalene Quantification using Nile Red Staining (M4455 Version)

$$
\text { Sebastian ST Triesch }{ }^{1}
$$

${ }^{1}$ Institute for Synthetic Microbiology, HHU Düsseldorf

$$
\text { Axmann Lab M4455 - Synthetische Biologie und Biotechnologie }
$$

\section{Sebastian ST Triesch}

Institute for Synthetic Microbiology, HHU Düsseldorf

\section{ABSTRACT}

\section{This protocol is under development!}

Nile Red is a fluorescent dye that stains selectively hydrophobic substances. We assume that squalene accumulates in the cell membrane or in lipid vesicles where it can be stained by the dye.

\section{GUIDELINES}

This protocol is under development and for teaching purposes only!

\section{MATERIALS}

\section{MATERIALS}

88 Nile Red Sigma Aldrich Catalog \#N3013 SIGMA

88 DMSO Sigma Aldrich Catalog \#D1435

BEFORE START INSTRUCTIONS

This protocol is under development and for teaching purposes only!

\title{
Culture Sampling
}

1 Sample 1-2 $\mathbf{m l}$ Synechocystis culture, measure its OD at $750 \mathrm{~nm}$ and adjust it to $\mathbf{2} \mathbf{~ m l ~ o f ~ O D ~}$ 
$(750 \mathbf{n m})=\mathbf{0 . 5}$ in BG-11 media. Split your adjusted culture in $\mathbf{2 x} \mathbf{1} \mathbf{~ m l}$. One portion will be stained with Nile Red, the other will serve as a negative control.

\section{Staining}

2 Stain one portion of the previously adjusted culture with $\mathbf{c}_{(\text {final })} \mathbf{=} \mathbf{5} \mathbf{\mu g} / \mathbf{m l ~ N i l e ~ R e d ~ i n ~ D M S O . ~}$ Add the same volume of DMSO to the unstained control samples.

\section{Safety information}

Wear gloves and a lab coat when working with DMSO as it stains skin and cloth.

\section{Washing}

3 Incubate your culures for $60 \mathrm{~min}$ in the dark.

01:00:00 Incubation

$4 \quad$ Centrifuge all samples for $\mathbf{5}$ min at $8000 \mathbf{x g}$.

(7) 00:05:00 Centrifugation

5 Carefully discard the supernatant by pipetting. Avoid resuspending the pellet or losing cells! Try removing as much supernatant as possible.

6 Thoroughly resuspend all pellets in $1 \mathrm{ml}$ BG-11 media, centrifuge again ( $5 \mathrm{~min}$ at $8000 \mathrm{xg}$ ), discard the supernatand and again resuspend the pellet in 1 ml BG-11 media.

\section{0:05:00 Centrifugation}

7 Optional: Keep the removed supernatant to check the quantity of free Nile Red in the supernatant! Ideally, you can repeat the washing steps until no more Nile Red is present in the supernatant. 


\section{Data Gathering}

8 Fill each $200 \mu \mathrm{l}$ of your cultures in 96 well plate's wells. Your sample volume should allow four wells per sample.

9 Measure each well's $\mathrm{OD}(750 \mathrm{~nm})$ in the plate reader.

10 Measure Nile Red fluorescence. Use following wavelenghts: Excitation: 510 nm/Emission: $660 \mathrm{~nm}$

11 Analyze your data: Normalize each well's fluorescence to the respective OD and subtract fluorescence without Nile Red from fluorescence with Nile Red. Compare your results to wild type Nile Red stained fluorescence. 